\title{
Tos ferina: revisión clínica a propósito de un caso
}

\author{
J. Olivares Ortiza , M. Bueno Campaña ${ }^{b}$ \\ aMIR-Pediatría. Hospital Universitario Fundación Alcorcón.Alcorcón, Madrid. España. \\ bUnidad de Pediatría. Hospital Universitario Fundación Alcorcón. Alcorcón, Madrid. España.
}

\section{Resumen}

La tos ferina es una enfermedad contagiosa que afecta en la actualidad a lactantes menores de seis meses y a adolescentes o adultos jóvenes, debido a una inmunidad vacunal limitada. Cursa clínicamente en varias fases, siendo el periodo de contagio la fase catarral inicial y las dos primeras semanas de la fase paroxística. En los lactantes pequeños puede presentarse de forma atípica, con pausas de apnea sin tos Ilegando a ser un cuadro potencialmente grave, por lo que pueden requerir ingreso. El diagnóstico es clínico, dadas las dificultades técnicas para la confirmación microbiológica. El germen responsable es Bordetella pertussis, aunque existen cuadros clínicos similares producidos por otros microorganismos, tanto virus como bacterias. El tratamiento antibiótico recomendado es con azitromicina tanto para el caso como para los contactos. No se ha demostrado que los tratamientos adyuvantes sean útiles.

Palabras clave: Tos ferina. Síndrome pertusoide. Profilaxis. Tratamiento.

Whooping cough: a case report and clinical review

\section{Abstract}

Whooping cough is an infectious disease that affects currently to infants less than six months old and teenagers or young adults, due to a limited vaccine efficacy. The clinical course has several phases, being the period of infection the initial catarrhal stage and the first two weeks of the paroxysmal phase. Young infants can have an atypical presentation with apnoea pauses without cough, becoming a potentially serious disease that may require admission. The diagnosis is clinical due to the technical difficulties in the laboratory confirmation. The responsible organism is Bordetella pertussis, although similar clinical symptoms can be produced by other micro-organisms, both viruses and bacteria. The recommended antibiotic is azithromycin for both the case and contacts. Complementary treatments have not proven useful.

Key words: Pertussis. Whooping Cough. Prophylaxis. Treatment. 


\section{Introducción}

La tos ferina es una enfermedad contagiosa que ha experimentado un aumento de prevalencia en los últimos años, debido a un cambio epidemiológico por el cual se afectan más adultos jóvenes y adolescentes, que son fuente de infección para lactantes, en los cuales la enfermedad puede ser grave.

\section{Caso clínico}

Lactante de 47 días sin antecedentes de interés que acude a la consulta de su pediatra por un cuadro de tos y mucosidad nasal de cuatro días de evolución, habiéndose hecho la tos más persistente en forma de accesos repetidos en los que le han observado cianosis perioral y que ocasionalmente terminan con un vómito; se encuentra afebril y con una reducción en la ingesta de leche materna.

En la exploración física se objetiva un buen estado general, con buenas coloración y perfusión periférica, sin signos de dificultad respiratoria y auscultación cardiopulmonar normal; se decide dejar en observación, durante la cual se objetiva uno de estos episodios en los que presenta un acceso con varios golpes de tos durante la espiración y con una inspiración ruidosa en forma de gallo al finalizar el acceso, acompañado de cianosis facial.

Se decide ingresarlo bajo la sospecha de síndrome pertusoide, con monitorización continua de saturación de oxígeno, frecuencia cardiaca y respiratoria. Durante su ingreso aumenta la frecuencia de los accesos de tos de tipo pertusoide, se objetiva repercusión en la frecuencia cardiaca y la saturación de oxígeno durante dichos accesos e, incluso, en los periodos intercríticos presenta pausas de apnea que se recuperan con estimulación, por lo que se le administra oxigenoterapia con gafas nasales y también sueroterapia intravenosa hasta que recupera una alimentación enteral suficiente; se trata con azitromicina al paciente y a los contactos cercanos.

Durante su ingreso hospitalario, se realiza un estudio serológico para Bordetella pertussis que resulta negativo tanto en la determinación inicial como en la siguiente, un mes después.

\section{Discusión}

La tos ferina es una enfermedad muy contagiosa, con una tasa de infección subclínica del $80 \%$ para aquellos individuos que han tenido una exposición intensa y que estaban previamente inmunizados $^{1,2}$. El periodo de contagio es la fase catarral inicial y las dos primeras semanas de la fase paroxística (hasta seis semanas en el caso de niños no imunizados) $)^{2}$. Ni la vacunación ni la infección 
natural consiguen una inmunidad completa. Del total de los casos, el 67\% aparece en adolescentes y adultos ${ }^{2}$, que son la principal fuente de infección para los niños ${ }^{3}$; en la actualidad existen dos picos de incidencia de la tos ferina: en los niños menores de cinco meses y en los mayores de diez años.

La duración de la inmunidad es limitada, de unos 15 años con la infección natural $^{1}$ y de 5-6 años con la vacunación ${ }^{4}$; la eficacia de la vacuna también se va perdiendo con el paso del tiempo; así, a los cuatro años es de un $84 \%$, y a los siete años es del $46 \%{ }^{5}$. La vacunación requiere un mínimo de dos dosis para ser eficaz ${ }^{6}$; por tanto, son los lactantes de menos de cuatro meses los de mayor riesgo. El principal origen de la infección en niños son los adultos que conviven con ellos, sobre todo los padres?.

Bordetella pertussis es el germen responsable de todos los casos de tos ferina epidémica y de la mayoría de las esporádicas. $B$. parapertussis es responsable de algunos casos de tos ferina esporádica, y B. bronchiseptica produce infecciones en inmunodeprimidos.

El síndrome pertusoide es un cuadro similar a la tos ferina, pero generalmente más leve y producido por otros gérmenes (Mycoplasma pneumoniae, Chlamydophila pneumoniae, Chlamydia tracho- matis, Ureaplasma urealyticum, virus respiratorio sincitial, adenovirus, Haemophilus parainfluenzae, citomegalovirus [CMV] y enterovirus).

El cuadro clínico clásico de la tos ferina cursa en varias fases: periodo de incubación de 7-10 días; fase catarral (1-2 semanas) indistinguible de cualquier infección respiratoria de vías altas, sin fiebre y con una tos progresivamente más intensa; fase paroxística (2-4 semanas), con accesos de tos con 5-10 golpes en cada espiración seguidos de un estridor inspiratorio característico conocido como gaIlo. Tras el acceso es frecuente expulsar una mucosidad espesa, filante o un vómito alimentario. Durante el paroxismo, la cara se muestra abotargada, cianótica, con hemorragias conjuntivales y protusión de la lengua, esto es lo que se conoce como facies pertusa. Entre paroxismos, el niño está asintomático. Posteriormente se sigue de una fase de convalecencia (1-3 semanas) en durante la cual las crisis de tos se van haciendo menos frecuentes y más leves, hasta que desaparecen.

En los menores de tres meses, el cuadro puede ser más grave y prolongado, la fase catarral puede pasar desapercibi$\mathrm{da}$, los accesos de tos son menos llamativos y sin gallo y es posible ver episodios de apnea o cianosis sin tos ${ }^{1}$. 
Las complicaciones más graves son la neumonía por Bordetella o por sobreinfección bacteriana (S. aureus, S. pneumoniae) $)^{2}$, esta última aparece en un $5 \%$ de los casos y es la causa del $90 \%$ de las muertes por tos ferina; $y$ por otro lado la encefalopatía tosferinosa (1/100 000 casos), con convulsiones y alteración del nivel de conciencia.

La necesidad de ingreso en Unidades de Cuidados Intensivos y de ventilación mecánica es más frecuente en menores de tres meses y se debe a insuficiencia respiratoria secundaria a apnea y a la neumonía bacteriana secundaria².

Se ha descrito el síndrome de la tos ferina maligna, que se da en menores de tres meses y se caracteriza por una reacción leucemoide (> 100000 leucocitos) con linfocitosis y bronconeumonía con hipoxemia e hipertensión pulmonar, que tiene una mortalidad del $75 \%$.

Dadas las dificultades técnicas y el retraso en los resultados del diagnóstico microbiológico, en la práctica el diagnóstico es fundamentalmente clínico.

Desde el punto de vista microbiológico, se puede realizar un cultivo que demuestre la presencia del germen en el aspirado nasofaríngeo profundo, mediante una torunda flexible de dacrón o de alginato cálcico, manteniéndola durante 15-30 segundos. Para la conserva- ción de la muestra durante un periodo de cuatro días se puede emplear el caldo de cultivo de Stainer-Scholte o el medio semisólido de Regan-Lowe². Dada la complejidad técnica del cultivo y puesto que finalmente la sensibilidad de la prueba es de menos del $50 \%$, se desaconseja su uso rutinario. Otra opción de diagnóstico microbiológico es la inmunofluorescencia directa (IFD), que puede hacerse sobre el cultivo o bien directamente en la muestra nasofaríngea, si bien es muy dependiente de la experiencia del laboratorio, alcanzando una sensibilidad del $60 \%$ y una especificidad del $90 \%$ en las condiciones óptimas ${ }^{1}$. El diagnóstico serológico se realiza mediante la detección en suero de IgG frente a toxina pertusoide (TP) y hemaglutinina filamentosa (HAF), que son detectables aproximadamente hasta cinco años después de la infección. Los anticuerpos dirigidos frente a TP son especificos para $B$. pertussis y se consideran marcadores serológicos de la infección de elección en niños. La detección de IgA se ha utilizado en algunos países para la definición de caso, pero no mejora el rendimiento del diagnóstico serológico frente a IgG y es más útil en niños mayores y adultos porque en niños pequeños, debido a su inmadurez inmunológica, los niveles de IgA en suero pueden ser indetectables. Para la confirmación 
diagnóstica sería necesario demostrar una seroconversión con un aumento en dos o cuatro veces del nivel de anticuerpos en una muestra convaleciente frente a una muestra inicial previa, lo cual no es posible en la práctica habitual. Se intenta utilizar determinaciones únicas de IgG TP para el diagnóstico serológico de la infección, para lo cual es necesario disponer de puntos de corte respecto a la población general, que de momento no están disponibles. En lactantes, se considera diagnóstica la existencia de títulos altos de IgG TP en una sola muestra en fase aguda y en ausencia de anticuerpos maternos ${ }^{3}$. Por último, la técnica de la reacción en cadena de la polimerasa (PCR) es la idónea, con una sensibilidad del $65-99 \%$ y una especificidad del 90 $100 \%$, mejores que las del resto de las técnicas.

La tos ferina es una enfermedad de declaración obligatoria (EDO), por lo que una vez diagnosticada se debe dar parte a los servicios de salud pública locales.

Se plantea el diagnóstico diferencial con síndrome pertusoide, recidivas clínicas de una tos ferina previa o bronquiolitis en los lactantes.

Ante la sospecha clínica de un caso de tos ferina, tanto en las consultas de Atención Primaria como en los Servicios de Urgencias Pediátricas, se debe valorar la necesidad de ingreso hospitalario (tabla 1).

Todo paciente ingresado por tos ferina debe ser monitorizado, se debe asegurar la adecuada nutrición e hidratación del niño, pudiendo ser necesaria la sueroterapia intravenosa, la alimentación a través de sonda nasogástrica o incluso la alimentación parenteral en los niños que no ganan peso, siendo esta la última opción ${ }^{2,8}$.

Es necesario el aislamiento respiratorio ${ }^{9}$ hasta que se hayan cumplido cinco días de tratamiento antibiótico efectivo o hayan pasado tres semanas desde el inicio de los síntomas.

El tratamiento antibiótico tiene dos objetivos: disminuir la gravedad de los síntomas $^{10}$ si el tratamiento se inicia dentro de los primeros siete días de clínica, y reducir el riesgo de transmisión ${ }^{11}$ eliminando el germen de la nasofaringe.

Se recomienda el tratamiento antibiótico según se indica en las tablas 2 y 3.

\begin{tabular}{l}
\hline Tabla 1. Criterios de ingreso $^{8}$ \\
\hline - Edad menor de seis meses \\
\hline - Presentación clínica de gravedad: \\
- Dificultad respiratoria \\
- Cianosis o apnea sin tos \\
- Convulsiones \\
- Neumonía \\
\hline - Imposibilidad para la alimentación \\
\hline
\end{tabular}


Tabla 2. Indicaciones de tratamiento antibiótico

- Sospecha clínica con confirmación bacteriológica o sin ella

- Diagnóstico microbiológico confirmado, aunque esté asintomático en el momento de la confirmación ${ }^{8}$

- Cuadro clínico de más de 21 días de duración pero permanecen en contacto con personas de alto riesgo (menores de cuatro meses, inmunodeficiencias, enfermedades crónicas, embarazadas en el tercer trimestre de gestación) $)^{12}$

Las pautas de tratamiento se describen res de un mes se excluye la claritromicina en la tabla 3 (recomendaciones de los porque se han registrado casos de esteCenters for Disease Control and Preven- nosis hipertrófica del píloro, y también el tion $\left.[C D C] 2005^{19}\right)$. Si no se pueden ad- TMP-SMZ, por el riesgo de kernicterus ministrar macrólidos, se puede utilizar el en los menores de dos meses.

cotrimoxazol (TMP-SMZ) en los mayores Los tratamientos coadyuvantes, incluide dos meses de edad. Los betalactámi- dos broncodilatadores, corticoides y ancos no son útiles en el tratamiento de la titusígenos, no han demostrado benefiinfección por Bordetella ${ }^{13}$. En los meno- cio en pacientes con tos ferina ${ }^{14}$. Por

Tabla 3. Recomendaciones de tratamiento de la tos ferina $(C D C, 2005)^{19}$

\begin{tabular}{|c|c|c|c|c|}
\hline Edad & Azitromicina & Eritromicina & Claritromicina & Cotrimoxazol \\
\hline$<1$ mes & $\begin{array}{l}\text { - Recomendado } \\
\text { - } 10 \mathrm{mg} / \mathrm{kg} / \text { día, } \\
\text { dosis única, } \\
5 \text { días }\end{array}$ & $\begin{array}{l}\text { - Si no es posible } \\
\text { con azitromicina } \\
\text { 40-50 mg/kg/ } \\
\text { día, en } 4 \text { dosis, } \\
14 \text { días }\end{array}$ & No recomendado & $\begin{array}{l}\text { Contraindicado } \\
\text { en menores de } \\
2 \text { meses }\end{array}$ \\
\hline $1-5$ meses & $\begin{array}{l}10 \text { mg/kg/día, } \\
\text { dosis única, } 5 \text { días }\end{array}$ & $\begin{array}{l}\text { 40-50 mg/kg/día, } \\
\text { en } 4 \text { dosis, } 14 \text { días }\end{array}$ & $\begin{array}{l}15 \text { mg/kg/día, } \\
\text { en } 2 \text { dosis, } \\
7 \text { días }\end{array}$ & 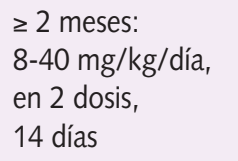 \\
\hline $\begin{array}{l}\geq 6 \text { meses } \\
\text { y niños }\end{array}$ & $\begin{array}{l}10 \text { mg/kg/día, } \\
\text { dosis única día 1; } \\
5 \mathrm{mg} / \mathrm{kg} / \text { día, días } \\
2 \text { a } 5\end{array}$ & $\begin{array}{l}\text { 40-50 mg/kg/día } \\
\text { (máximo } 2 \text { g/día), } \\
\text { en } 4 \text { dosis, } 14 \text { días }\end{array}$ & $\begin{array}{l}15 \text { mg/kg/día } \\
\text { (máximo } 1 \mathrm{~g} / \text { día), } \\
\text { en } 2 \text { dosis, } 7 \text { días }\end{array}$ & $\begin{array}{l}\text { 8-40 mg/kg/día, } \\
\text { en } 2 \text { dosis, } 14 \text { días }\end{array}$ \\
\hline Adultos & $\begin{array}{l}500 \mathrm{mg} \text {, dosis } \\
\text { única, día 1; } \\
250 \mathrm{mg} \text { días } 2 \text { a } 5\end{array}$ & $\begin{array}{l}500 \mathrm{mg} / 6 \text { horas, } \\
14 \text { días }\end{array}$ & $\begin{array}{l}500 \mathrm{mg} / 12 \text { horas, } \\
7 \text { días }\end{array}$ & $\begin{array}{l}160-800 \mathrm{mg} / 12 \\
\text { horas, } 14 \text { días }\end{array}$ \\
\hline
\end{tabular}


tanto, no hay evidencia suficiente para recomendar ninguno de ellos, y en algún caso se recomienda no usarlos por sus efectos adversos. El salbutamol, ya sea inhalado o por vía oral, se ha probado en dos estudios con un total de 34 pacientes en los que no se ha demostrado diferencia significativa con el grupo contro| ${ }^{15,16}$, aunque algunos autores recomiendan hacer un ensayo terapéutico con betaagonistas inhalados en caso de compromiso respiratorio. Con respecto a los corticoides, existe un estudio con 11 pacientes en tratamiento con dexametasona oral en el que no se demuestra diferencia significativa ${ }^{17} ;$ existen datos individuales que sugieren un posible beneficio clínico con el uso de corticoides, aunque no se recomiendan de forma rutinaria. Los antitusígenos como la codeína no solo no son eficaces, sino que están contraindicados por su efecto sedante ${ }^{8}$.

En cuanto al uso de inmunoglobulinas específicas para antígenos de Bordetella pertussis, un estudio demostró una disminución en la frecuencia de accesos de $\operatorname{tos}^{18}$, pero no hay datos suficientes para recomendar su uso habitual.

El uso de tratamiento antibiótico profiláctico postexposición a los contactos en los primeros 21 días de clínica del caso inicial puede prevenir el desarrollo de los síntomas; pasado este tiempo la indicación no está clara la indicación y habrá que valorar el beneficio-riesgo individual ${ }^{8}$.

Las indicaciones de tratamiento antibiótico profiláctico alcanzan a todos los contactos cercanos (exposición cara a cara a menos de 1 metro del paciente, contacto directo con las secreciones respiratorias, se comparte un espacio cerrado próximo al paciente durante al menos una hora ${ }^{19}$ ) incluidos los cuidadores de la guardería y miembros de la familia, independientemente de su estado vacunal ${ }^{9}$. Siguiendo la misma pauta de tratamiento antibiótico que para los pacientes y dentro de los primeros 21 días de clínica del paciente caso.

Para los convivientes y contactos íntimos menores de siete años se administrará una dosis adicional de DTPa si han recibido cuatro dosis. A los que han recibido menos de cuatro dosis de DTPa se les revacunará a intervalos de cuatro semanas, con las dosis que les falten hasta completar el calendario vacunal. En los de siete o más años, adolescentes y adultos puede administrarse Tdpa (con carga antigénica reducida de difteria y tos ferina) $)^{19}$.

Dado que la principal fuente de infección son los adolescentes y adultos, el Comité Asesor de Vacunas de la Asocia- 
ción Española de Pediatría recomienda la vacunación a los 14 años con Tdpa en lugar de la Td actual ${ }^{20}$.

Algunos autores se plantean la vacunación de mujeres embarazadas en el último trimestre, ya que podría dar protección al lactante hasta que reciba la primera dosis de la vacuna. Pero se ha visto que los anticuerpos transferidos por la madre son indetectables a los 2-6 meses $^{21}$ y además podrían interferir con la respuesta inmunógica tras la vacunación ${ }^{22}$. La vacunación en la embarazada solo se hará en situaciones de alto riesgo' ${ }^{1}$.

Se han realizado algunos estudios con vacunación en recién nacidos con resultados heterogéneos y no concluyentes, en los que se encuentran un nivel elevado de anticuerpos de forma precoz a los 2-3 meses, pero niveles más bajos a los 7-8 meses, con respecto a la pauta vacunal habitual ${ }^{23-25}$. En la actualidad no se recomienda la vacunación neonatal. Los niños que han pasado la tos ferina deben con- tinuar con su calendario vacunal habitual. En resumen, la tos ferina es una enfermedad de prevalencia global descendiente pero con un cambio en su patrón epidemiológico que ha hecho aumentar su presencia en adultos jóvenes vacunados en su infancia y en lactantes pequeños antes de que su vacunación sea efectiva, y es en estos últimos en los que se dan con cierta frecuencia cuadros que pueden llegar a ser graves. Por tanto, es conveniente tener en cuenta esta patología en el conjunto de las infecciones respiratorias que se ven habitualmente en las consultas. Las dificultades en el diagnóstico microbiológico hacen que el diagnóstico diferencial entre las distintas causas del síndrome pertusoide deba basarse principalmente en la clínica. En cualquier caso, la actitud terapéutica habrá de ser la misma y, por tanto, la confirmación microbiológica, en general, no es necesaria.

\section{Bibliografía}

1. Moraga FA, Campins M. Tos ferina. En: Delgado Rubio A. Enfermedades infecciosas en Pediatría, 1. ${ }^{a}$ ed. Madrid: McGraw-Hill/Interamericana de España, SAU; 2009. p. 73-80.

2. Long SS. Tos ferina (Bordetella pertussis y Bordetella parapertussis). En: Kliegman RM, Behrman
RE, Jenson HB, Stanton BF. Nelson Tratado de Pediatría, 18. a ed. Barcelona: Elsevier; 2009. p. 1178-82.

3. Sanz JC, de Ory F. Diagnóstico de laboratorio de tos ferina. Papel de la serología. Enferm Infecc Microbiol Clin. 2002;20(5):212-8.

4. Wendelboe A, Van Rie A, Salmaso S, Englund JA. Duration of inmunity against pertussis after 
natural infection or vaccination. Pediatr Infect Dis J. 2005;25:S58-S61.

5. Jenkinson D. Duration of effectiveness of pertussis vaccine. Br Med J. 1988;286:612-4.

6. Olin P, Gustafsson L, Barreto L, Hessel L, Rie $\mathrm{AV}$, Bogaerts $\mathrm{H}$ et al. Declining pertussis incidence in Sweden following the introduction of acellular pertussis vaccine. Vaccine. 2003;21:2015-21.

7. Forsyth K, Tan T, König CH, Caro JJ, Plotkin $\mathrm{S}$. Potential strategies to reduce the burden of pertussis. Pediatr Infect Dis J. 2005;24:S69-S74.

8. Yeh S. Treatment and prevention of BordeteIla pertussis infection in infants and children. Uptodate 18.3 [en línea] [actualizado el 27/09/2010; consultado el 07/03/2011]. Disponible en www. uptodate.com

9. American Academy of Pediatrics. Pertussis (whooping cough). En: Pickering LK (ed.). Red Book: 2009 report of the committee on infectious diseases, 28th ed. Elk Grove Village, IL: American Academy of Pediatrics; 2009. p. 504.

10. Bass JW. Pertussis: current status of prevention and treatment. Pediatr Infect Dis J. 1985;4:614.

11. Bortolussi $R$, Miller $B$, Ledwith $M$, Halperin S. Clinical course of pertussis in immunized children. Pediatr Infet Dis J. 1995;14:870-4.

12. Center for Disease Control and Prevention guidelines for the control of pertussis outbreaks. Atlanta, GA: Center for Disease Control and Prevention; 2000.

13. Trollfors B. Effect of erythromycin and amoxicillin on Bordetella pertussis in the nasopharynx. Infection. 1978;6:228-30.

14. Pillay V, Swingler G. Symptomatic treatment of the cough in whooping cough. Cochrane database Syst rev. 2003;CD00 3257.

15. Metsola J, Viljamen MK, Ruskanen O. Salbutamol in the treatment of whooping cough. Scand J Infect Dis Rev. 1986;18:593.
16. Krantz I, Norrby SR, Trollfors B. Salbutamol vs placebo for treatment of pertussis. Pediatr Infect Dis. 1985;4:638-40.

17. Roberts I, Gavin R, Lennon D. Randomizaed controlled trial of steroids in pertussis. Pediatr Infect Dis J. 1992;11:982-3.

18. Hewlett EL, Edwuards KM. Clinical practice. Pertussis not just for kids. N Engl J Med. 2005;352:1215-22.

19. Martínez Antón A, Ordóñez Sáez O, Ruiz Contreras J. Síndrome pertusoide (v.1/2007). En: Hernández Merino A (coord.). Guía-ABE. Infecciones en Pediatría. Guía rápida para la selección del tratamiento antimicrobiano empírico [en línea] [actualizado el 17/10/2007; consultado el 07/03/2011]. Disponible en www.guia-abe.es

20. Marès Bermúdez J, van Esso Arbolave $D$, Moreno-Pérez D, Merino Moína M, Álvarez García FJ, Cilleruelo Ortega MJ, y cols. Calendario de vacunaciones de la Asociación Española de Pediatría: recomendaciones 2011. An Pediatr (Barc). 2011;74:132.e1-132.e19.

21. Tiwani T, Murphy TV, Moran J. Recommended antimicrobial agents for the treatment and postexposure prophylaxis of pertussis: 2005 CDC guidelines. MMWR Recomm Rep. 2005; 54 (RR14);16.01.

22. Edwuard KM. Pertussis and important target for maternal immunization. Vaccine. 2003;21:3483-6.

23. Englund JA, Anderson EL, Reed GF. The effect of maternal antibody on serologic response and the incidence of adverse reactions after primary immunization with acellular and whole-cell pertussis vaccines combined with diphtheria and tetanus toxoids. Pediatrics. 1995;96:580-4.

24. Belloni C, De Silvestri A, Tinelli C, Avanzini MA, Marconi M, Strano F et al. Immunogenicity of a three-component acellular pertussis vaccine administered at birth. Pediatrics. 2003;111:1042-15. 
25. Halasa NB, O'Shea A, Shi JR, LaFleur BJ, Edwards KM. Poor inmune responses to a birth dose of diphteria, tetanus and acellular pertussis vaccine. J Pediartr. 2008;153:327-32.
26. Knuf $M$, Schmidt HJ, Wolter J, Schuerman $L$, Jacquet $J M$, Kieninger $D$ et al. Neonatal vaccination with acellular pertussis vaccine accelerates the acquisition of pertussis antibodies in infants. J Pediatr. 2008;152:655-60. 